\title{
Morphology of Amorphous Pockets in SiC Irradiated with 1 MeV Kr Ions
}

\author{
Xing Wang ${ }^{1}$, Laura Jamison ${ }^{2}$, Kumar Sridharan ${ }^{1,3}$, Paul M. Voyles ${ }^{2,3}$, Dane Morgan ${ }^{1,2,3}$ and Izabela \\ Szlufarska ${ }^{1,2,3}$ \\ 1. Department of Nuclear Engineering, UW-Madison, Madison, WI, USA. \\ 2. Materials Science Program, UW-Madison, Madison, WI, USA \\ 3. Department of Materials Science and Engineering, UW-Madison, Madison, WI, USA
}

Radiation-induced amorphization is one of the concerns with $\mathrm{SiC}$ as structural components for next-generation nuclear reactors. Numerous studies have been focused on the amorphization mechanisms under electron, neutron, and ion irradiation. Proposed mechanisms for ion-induced amorphization include nucleation and growth of amorphous regions, direct impact combined with stimulated amorphization at crystalline/amorphous (c/a) boundaries, and homogeneous amorphization due to defect accumulation [1]. However, it is still not clear which mechanism governs the amorphization process. Furthermore, current simulation and experimental results imply that the mechanism depends on the irradiation species and on the nano/microstructure of SiC.

Here, we use high-resolution transmission electron microscopy (HRTEM) to image amorphous pockets in partially amorphized $\mathrm{SiC}$ to elucidate the underlying amorphization mechanisms. In our experiment, $3 \mathrm{C}$-SiC samples (with $\mu \mathrm{m}$-sized grains) were irradiated in situ at $100^{\circ} \mathrm{C}$ with $1 \mathrm{MeV} \mathrm{Kr}$ ions using the IVEM-Tandem facility at the Argonne National Laboratory. The dose to amorphization was first determined based on the electron diffraction pattern. Then another sample was irradiated to $50 \%$ of the dose to amorphization. After the irradiation, the samples were investigated using HRTEM in a FEI Tecnai TF-30 TEM, operated at $300 \mathrm{kV}$. Two methods were used to draw the contour of the amorphous pockets. At lower magnification $(470 \mathrm{kx})$, the images were Fourier filtered to enhance the contrast between crystalline and amorphous regions. For higher magnification (800 kx), the c/a boundary was directly drawn on the image by hand.

HRTEM images (Fig.1(a) and Fig.2(a)) of the 50\% dose sample show the amorphization to be heterogeneous, which is consistent with previous studies [1,2]. Amorphous pockets, which are a few nm size, are separated by crystalline regions. The c/a boundaries are shown in Fig. 1(b) and 2(b). The dendrite-like morphology of the amorphous pockets is surprising and qualitatively different from the larger ( $25 \mathrm{~nm}$ long, $10 \mathrm{~nm}$ wide) island-shaped amorphous pockets in $0.5 \mathrm{MeV}$ Si ion irradiated $\mathrm{SiC}$ [2]. We hypothesize that the dendritic morphology arises from the combined effects of direct amorphization, defect-stimulated amorphization at c/a boundary, and a 2D projection effect in HRTEM images. To test this hypothesis, we developed a coarse-grained model of irradiation and used it to simulate the morphology of the observed c/a boundaries. In the simulation, $\mathrm{SiC}$ was represented as a 3D matrix of $256^{3}$ nodes (lattice points). The distance between lattice points was taken to be $2.18 \AA$ and the size of the simulated $\mathrm{SiC}$ sample was $55.8 \mathrm{~nm}^{3}$, so that the density of nodes was equal to the density of atoms in SiC. Direct amorphization was modeled by introducing ellipsoidal amorphous regions (with axes lengths $a=1.31 \mathrm{~nm}, \mathrm{~b}=\mathrm{c}=0.65 \mathrm{~nm}$ ) at random positions, simulating the effect of single ion collisions. Regions that were amorphous stayed amorphous under further irradiation. Based on the data from electron irradiation, a defect-stimulated growth rate of the c/a boundary was estimated to be $8.72 \times 10^{-4} \mathrm{~nm} /$ collision [3]. After 600 collisions, the 3D matrix was projected along the $\mathrm{z}$-direction to 
create the final 2D images. The simulated image is shown in Fig. 1(c), which is qualitatively consistent with the dendritic shape of the amorphous pockets from the experiment (Fig. 1(b)).

We have also determined the effect of grain boundaries (GBs) on the degree (fraction) of amorphization as observed in HRTEM images. We found the amorphization fraction is larger near GBs ( $56 \%)$ than away from GBs $(\sim 42-44 \%)$. This results seems surprising given that GBs are expected to provide an effective sink for radiation induced defects. However, our observation can be explained by the interstitial-starvation phenomenon predicted in our earlier rate theory models [3]. The presence of interstitial starvation suggests that point defects interact with the GBs. Consequently these results imply in addition to the direct amorphization, $\mathrm{Kr}$ ions amorphize $\mathrm{SiC}$ indirectly by accumulation of point defects [4].

\section{Reference}

[1] W.J. Weber, Nucl. Instrum. Meth. B, 166 (2000) p. 98-106.

[2] L.L. Snead, S.J. Zinkle et al, Nucl. Instrum. Meth. B, 141 (1998) p. 123-132.

[3] N. Swaminathan, D. Morgan, I. Szlufarska, Phys. Rev. B, 86 (2012) p. 214110

[4] This research is supported by the US Department of Energy, Office of Basic Energy Sciences (grant

DE-FG02-08ER46493).
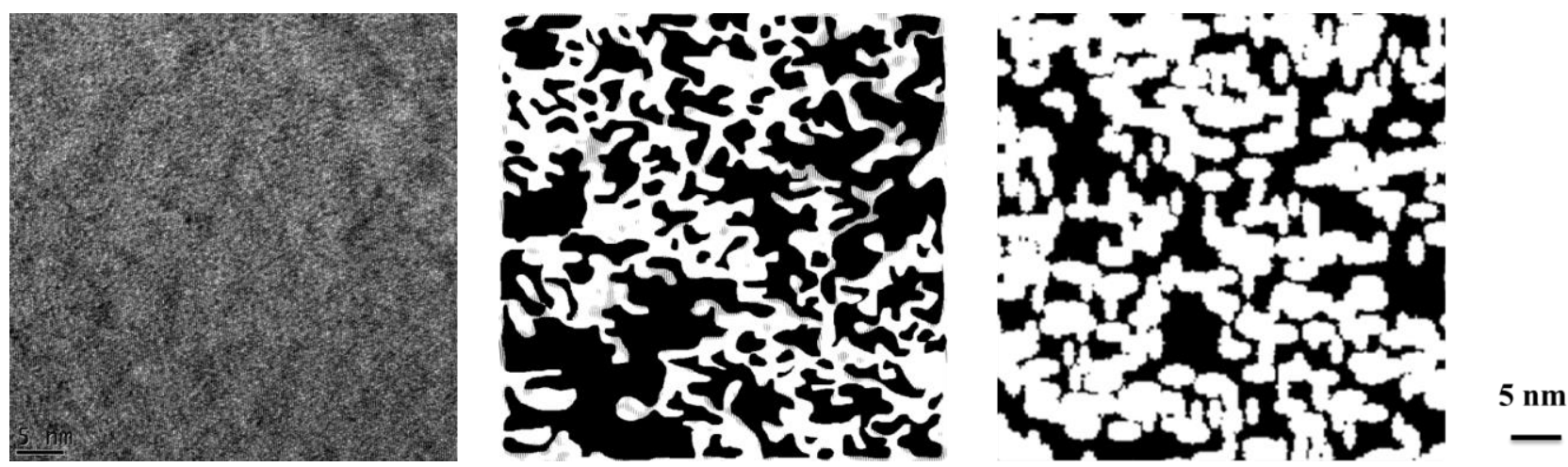

Figure 1. (a) HRTEM image of $\mathrm{SiC}$ irradiated up to $50 \%$ of the dose to amorphization at Mag=470 $\mathrm{kx}$. (b) Fourier filtered image. (c) Simulated images. (In (b) and (c), black represents crystalline and white represents amorphous.)
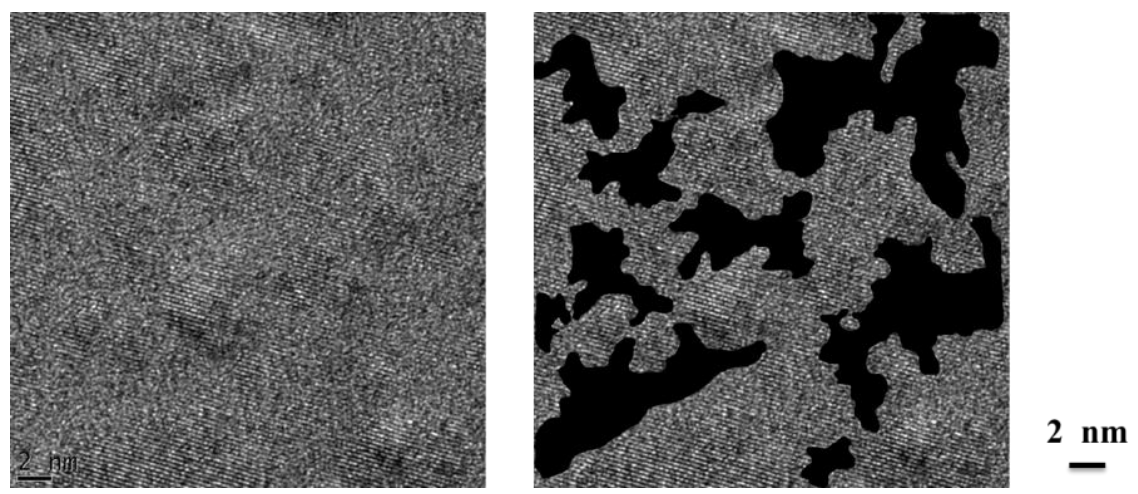

Figure 2. (a) HRTEM image of $\mathrm{SiC}$ irradiated up to $50 \%$ of the dose to amorphization at Mag=800 $\mathrm{kx}$. (b) Directly drawn contour of amorphous pockets. (In (b), black regions represent amorphous pockets.) 